\title{
Design and Development of Novel Miniaturized UHF RFID Tags on Ultra-low-cost Paper-based Substrates
}

\author{
Li Yang, Serkan Basat, Amin Rida, and Manos M. Tentzeris \\ Georgia Electronic Design Center, School of Electrical and Computer Engineering, \\ Georgia Institute of Technology, Atlanta, GA 30332-0250, USA
}

E-mail: gth772t@mail.gatech.edu

\begin{abstract}
In this paper, an overview of design requirements and novel approaches for improved performance UHF radio frequency identification (RFID) tags is presented. Two matching techniques, inductively coupled structure and serial stub structure are discussed. Different antenna configurations are proposed, focusing on low-profile, low polarization mismatch and high directivity. Paper substrates, that could potentially utilize inkjet printing techniques, are also investigated for the realization of ultra-low-cost RFID tags.
\end{abstract}

Index Terms - RFID, Antennas, inductively coupled matching, inkjet printing, low profile radiators, paper, polarization diversity, serial stub matching.

\section{INTRODUCTION}

The demand for flexible RFID tags has rapidly increased due to the requirements of automatic identification in various areas, such as item-level tracking, access control, electronic toll collection and vehicle security [1]. Compared with the lower frequency tags (LF and HF bands) already suffering from limited read range (1-2 feet), RFID tags in UHF band see the widest use due to their higher read range (over 10 feet) and higher data transfer rate [2]. Two major challenges exist in today's RFID technology advancing toward the practical level. One is the design of tag antennas with higher efficiency and better impedance matching for IC chips with typically high capacitive reactance [3]. This is essential to maximize the RFID system performance. Another major challenge is the realization of ultra-low-cost RFID tags. Economical applications require the cost of individual tags drop down to one or two cents [4].

In this paper, we briefly report various novel approaches in three aspects to tackle these challenges, namely realization of matching techniques, design of antenna structures and development of printed RFID tags. Examples are supplemented with modeling and simulation results.

\section{MATCHING TECHNIQUES}

Most available commercial RFID tags are passive, due to cost and fabrication requirements. For such tags, the antenna translates electromagnetic waves from the reader into power supplied to the chip. Thus, a conjugate impedance matching between the antenna and the tag chip is highly essential to power up the chip and maximize the effective range. For years, antennas have been designed primarily to match either $50 \Omega$ or $75 \Omega$ loads. However, RFID chips primarily exhibit complex input impedance, making matching extremely challenging. While adding an external matching network is highly undesirable due to space constraints, two feed techniques, namely inductively coupled structure and serial stub configuration, provide solutions for an effective direct matching from ultra-compact tag antennas to highly capacitive RFID chips.

\section{A. Inductively Coupled Feed Structure}

A typical inductively coupled feed structure is shown in Fig. 1. The antenna consists of a feed loop and a radiating body. Two terminals of the loop are connected to the chip, and the feed is combined with the antenna body through mutual coupling.

The inductively coupled structure can be modeled as a transformer. Fig. 1 also depicts the equivalent lumped element model, where $\mathrm{R}_{\mathrm{rb}}$ and $\mathrm{R}_{\text {loop }}$ are the individual resistances of the radiating body and the

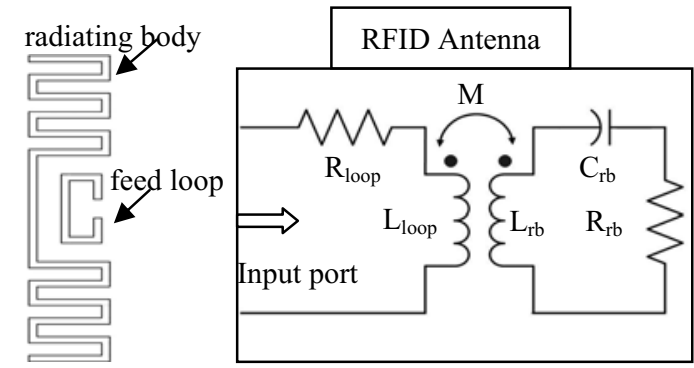

Rrb: radiation resistance

Crb: capacitance of the radiating body

Lrb: self-inductance of the radiating body

Lloop: self-inductance of the feed loop

M: mutual inductance

Rloop: resistance of the feed loop

Fig. 1. Configuration of inductively coupled RFID tag antenna and its lumped element model. 


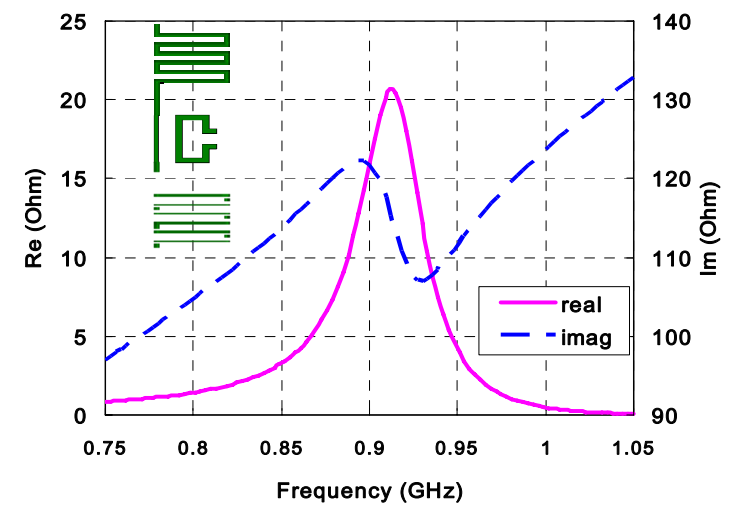

(a)

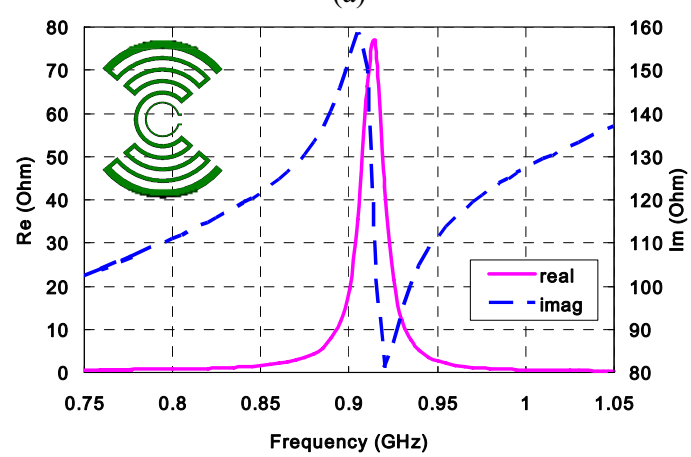

(b)

Fig. 2. Antenna impedance against frequency: (a) Target IC impedance 20-j113 $\Omega$. (b) Target IC impedance 73-j113 $\Omega$.

feed loop. $\mathrm{M}$ is the mutual inductance and $\mathrm{L}_{\text {loop }}$ is the self-inductance of the feed loop. At resonant frequency, the components of antenna input impedance can be predicted as

$$
\begin{aligned}
& R a=\frac{\left(2 \pi f_{0} M\right)^{2}}{R_{r b}}+R_{\text {loop }} \\
& X a=2 \pi f_{0} L_{\text {loop }}
\end{aligned}
$$

$\mathrm{R}_{\text {loop }}$ is typically small. Therefore, the resistance is mainly controlled by $\mathrm{M}$ and $\mathrm{R}_{\mathrm{rb}}$, and the reactance is dependent only upon $\mathrm{L}_{\text {loop }}$ [3]. In this way, the antenna input resistance $\mathrm{Ra}$ and input reactance $\mathrm{Xa}$ can be adjusted independently, and can be theoretically matched to arbitrary chip impedance.

Fig. 2(a) and (b) illustrate two inductively coupled feed antennas, aiming at low and high IC resistance, respectively, with capacitive reactance in both cases. It should be noted that the impedance response will become sharp to achieve high antenna input resistances in the second case, resulting in a narrow bandwidth which is not critical for most of the traditionally narrowband UHF RFID tags. An alternative feed structure that provides effective conjugate matching in a more broadband fashion, the serial stub, is preferred in this situation and will be presented in the next section.

\section{B. Serial Stub Feed Structure}

The topology of the serial stub feeding [2] is demonstrated in Fig. 3. In this design the overall matching network is designed to conjugately match a chip impedance of $Z_{\mathrm{IC}}=73-\mathrm{j} 113$. The resistive shorting stub and the double inductive stub constitute the overall matching network. The shorting stub mainly controls the resistive matching and the double inductive stub controls the reactive matching. The double inductive stub structure is composed of two inductive stubs to provide symmetry on both sides of the RFID tag. The IC is to be surface mounted on the bottom part of the double inductive stub which becomes the feeding point of the antenna

The input impedance of the simulated antenna design is shown in Fig. 4. As it can be observed the UHF RFID band of the serial stub is outside the antenna self-resonance peak at $750 \mathrm{MHz}$, resulting in a more flat impedance response against frequency. This yields an operational bandwidth of $13.5 \%$ compared with $1.5 \%$ bandwidth of the inductively coupled feed structure shown in Fig. 2(b). However, since serial stub feed antennas yield lower phase angles than inductively coupled ones, it is more preferred to cooperate with low reactance IC chips.

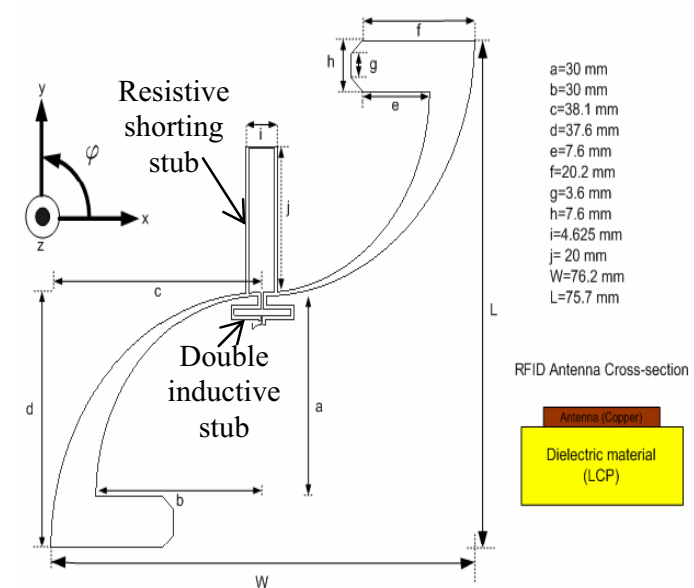

Fig. 3. RFID antenna structure showing serial stubs

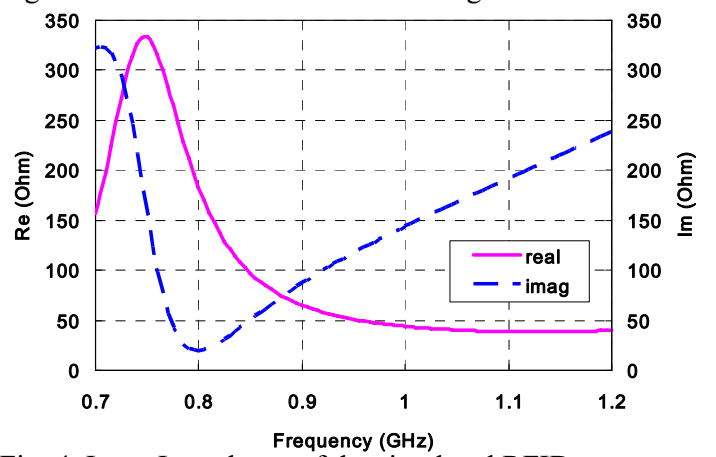

Fig. 4. Input Impedance of the simulated RFID antenna 


\section{ANTENNA STRUCTURES}

\section{A. Meander line}

RFID antenna is usually built to achieve halfwavelength resonance at first for efficiency optimization. This is approximately equal the maximum length, when the dipole antenna is stretched from one end to the other. In the UHF band, a half-wave dipole antenna is almost $16 \mathrm{~cm}$ in free space. For the purpose of reducing tag sizes, the meander line structure in Fig. 2(a) is an attractive choice. Arc-shape configuration is a modification of the meander line, as shown in Fig. 2(b). It exhibits smoother transition along the radiating arms. This class of antenna provides the largest size reduction (down to a quarter wavelength) at desired frequency at the expense of a slight decrease in gain (usually $5 \%$ lower) due to less effective radiating length.

\section{B. Dual polarization structure}

Antennas in UHF RFID tags are linearly (vertical or horizontal) polarized. In the presence of environmental reflections which cause the multipath effect, the transmitted/received plane waves undergo polarization direction changes. For instance, a vertically polarized transmitted wave can reach a tag at its blind spot, namely null in the radiation pattern. This causes the RFID tag not to be read. In order to prevent this, polarization diversity has to be utilized, requiring the use of both vertical and linear polarized antennas. For dipole antennas, the two antennas are oriented orthogonal to each other as shown in Fig. 5. These two antennas are identical in dimensions and shape, so that the identical signals arriving at these two different branches are in-phase and uncorrelated. This is critical for the demodulator in the IC that no phase difference occurs when same data is retrieved from the combined reception of the two antennas.

Fig. 6 shows a $3 "$ x 3" dual polarized antenna. The shorting stub that connects the top left legs (RF port) of the design both provides inductive conjugate matching and is also used to dc-short the two orthogonal dipole antennas. The bottom right legs are connected to ground to achieve signal ground excitation. The input impedance of this dual antenna is $20+\mathrm{j} 112.5 \Omega$ to match to an IC input impedance of $20-\mathrm{j} 113 \Omega$. The radiation efficiency is $98 \%$ since the current flow as shown in the figure adds up constructively for the far-field electromagnetic radiation, something that results in an optimized performance in terms of the read rage and environment versatility. The radiation pattern of the

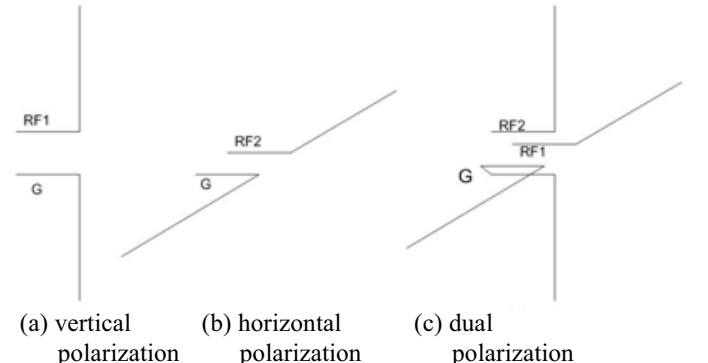

Fig. 5. Polarization definition in RFID antenna design
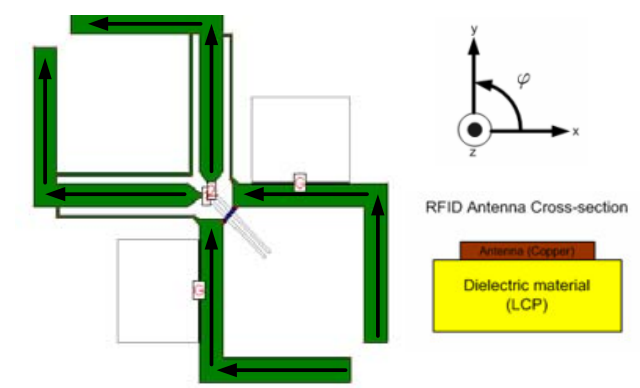

Fig. 6. Dual polarization antenna (arrows indicate current flow directions)

dual polarized antenna is omnidirectional in the $y-z$ plane with a maximum directivity of $2.25 \mathrm{dBi}$.

\section{Dual-body Configuration}

As it was reported before, the design of a high directivity RFID antenna is an effective method to increase the read range of a tag. However, the performance of most RFID antennas is constrained by their intrinsic dipole nature and a high directivity, especially for narrow-beamwidth conveyor belt applications, is very difficult to achieve. Adding another radiating body is a solution to this. A new topology, named dual-body configuration is presented in Fig. 7. Two meander line arms are placed in the same side of the feeding loop as shown in Fig. 7(a), and a directivity of $2.69 \mathrm{dBi}$ is achieved. In Fig. 7(b), two meander line arms are placed in each side of the feeding loop. In this case, the current directions are opposite along the arms and the radiation patterns cancel out each other in most of the directions. Thus, in this inductively coupled RFID antenna, the radiating energy is focused directionally in a dumbbell shape and a high directivity of $5.62 \mathrm{dBi}$ is observed with $79.9 \%$ radiation efficiency. In general, a highly increased effective range is expected to achieve with RFID antennas in such a configuration.

In addition, dual-body configuration can also be used to enhance the antenna bandwidth performance. Radiating bodies can resonate in adjacent frequencies by adjusting the length of each arm. In this way, an antenna can resonate at multi frequencies to enlarge 


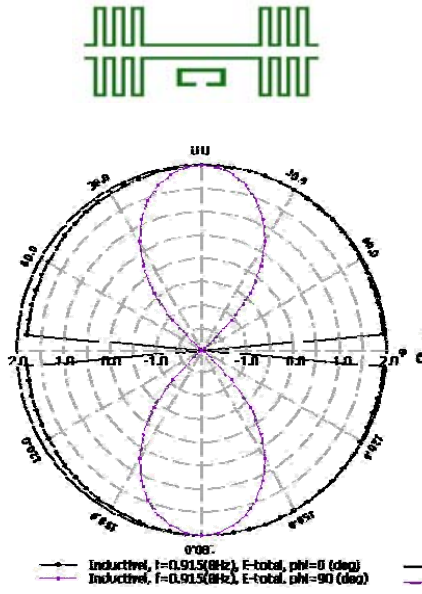

(a)

\section{땜ㄷํำ}

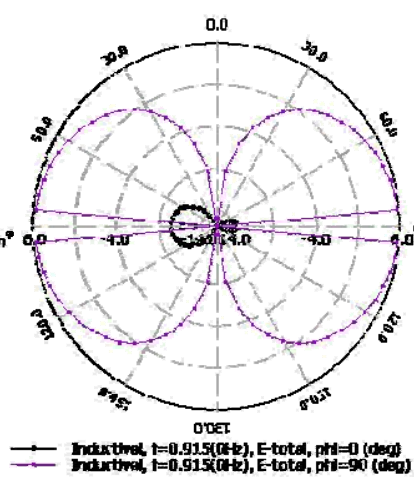

(b)
Fig. 7 Two dual radiating bodies RFID antennas with radiation patterns. (a) Directivity $=2.69 \mathrm{dBi}$, efficiency $=$ $86.8 \%$; (b) Directivity $=5.62 \mathrm{dBi}$, efficiency $=79.9 \%$.

bandwidth and make possible multiband/ multistandard RFID tags.

\section{RFID EMBEDDED IN/ON ORGANIC SUBSTRATES: PAPER}

Paper is considered as one of the best organic substrates for RFID applications. First of all, paper is environmentally friendly and can undergo large reel to reel processing. In terms of mass production and increased demand, this makes paper the lowest cost material made. Paper also has low surface profile with appropriate coating. This is very crucial since fast printing processes, such as conductive paste inkjet printing, can be used instead of metal etching techniques, something that will allow for the decrease of the cost down to a few cents for passive tags. In addition, paper is compatible with circuit printing by direct write methodologies. This is one of the biggest advantages of paper since active tags require additional modules like sensors and batteries to be mounted on or embedded in. A fast process like inkjet printing can be used efficiently to print these modules on or in the paper substrate. Paper can also host nanoscale additives (i.e. fire retardant textiles) and can be hydrophobic. Most importantly its dielectric constant $\varepsilon_{\mathrm{r}}(\sim 3)$ is close to air's (5-6\% power reflection). Electromagnetic power can still penetrate easily even if the RFID is embedded in the substrate.

Paper substrate can excellently handle high temperature treatment during the assembly process and the reliability/life time is very high compared to other substrates such as plastic. In addition to this, low thickness can be easily achieved with paper. For instance, adding a contactless smart label can significantly increase a document's overall thickness to encapsulate the IC. Plastic module needs $350 \mathrm{um}$ thickness with a surface of $7 \mathrm{~mm} \mathrm{x} 7 \mathrm{~mm}$; meanwhile, paper module only uses 150 um thickness with a surface less than $11 \mathrm{~mm}^{2}$ (equivalent to 2 sheets of $80 \mathrm{~g}$ paper) [5]. Paper can also be characterized easily for electrical properties using a ring resonator structure. Dielectric constant, $\varepsilon_{\mathrm{r}}$ and loss tangent, tan $\delta$ can be effectively accurately measured up to $40 \mathrm{GHz}$ [6]. At the conference, we will present an extensive set of theoretical and experimental results of paper-based RFID's.

\section{CONCLUSION}

In this paper, we presented a set of novel approaches for the design of miniaturized enhanced performance UHF RFID tags. Various design aspects have been outlined including effective compact conjugate matching techniques, dual polarization antenna structure and dual radiating bodies configuration. Modeling and simulation results have been reported with good agreement. Paper as a potential material for RFID substrates is also investigated to realize ultra-low-cost RFID tags, utilizing cheap inkjet printing techniques.

\section{REFERENCES}

[1] K. Finkenzeller, RFID Handbook, $2^{\text {nd }}$ ed., Wiley, 2004.

[2] S. Basat, S. Bhattacharya, A. Rida, S. Johnston, L. Yang, M.M. Tentzeris, and J. Laskar "Fabrication and Assembly of a Novel High-Efficience UHF RFID Tag on Flexible LCP Substrate" Proc. of the IEEE-ECTC Symposium, San Diego, June 2006

[3] H.-W Son and C.-S Pyo, "Design of RFID tag antennas using an inductively coupled feed," Electronics Letters, vol. 41, pp. 994 - 996, No. 18, Sept. 2005.

[4] V. Subramanian, J. M. Frechet, and etc., "Progress Toward Development of All-printed RFID Tags: Materials, Processes, and Devices," Proceedings of the IEEE, vol. 93, pp. 1330 - 1338, No. 7, July 2005.

[5] International Paper Knowledge Center paper RFID information [online], http://glossary.ippaper.com/ default.asp? $r e q=k n o w l e d g e / a r t i c l e / 489$

[6] D. C. Thompson, O. Tantot, H. Jallageas, G. E. Ponchak, M. M. Tentzeris, J. Papapolymerou, "Characterization of Liquid Crystal Polymer (LCP) Material and Transmission Lines on LCP Substrates From 30 to $110 \mathrm{GHz}$ "IEEE Trans. Microwave Theory and Tech., vol 52, issue 4, pp.1343-1352, April 2004 